\title{
Hijacking Cortical Motor Output with Repetitive Microstimulation
}

\author{
Darcy M. Griffin, ${ }^{1}$ Heather M. Hudson, ${ }^{1}$ Abderraouf Belhaj-Saif, ${ }^{1,2}$ and Paul D. Cheney ${ }^{1}$ \\ ${ }^{1}$ Department of Molecular and Integrative Physiology, University of Kansas Medical Center, Kansas City, Kansas 66160, and ${ }^{2}$ Unit of Physiology, \\ Department of Medicine, University of Fribourg, 1700 Fribourg, Switzerland
}

High-frequency repetitive microstimulation has been widely used as a method of investigating the properties of cortical motor output. Despite its widespread use, few studies have investigated how activity evoked by high-frequency stimulation may interact with the existing activity of cortical cells resulting from natural synaptic inputs. A reasonable assumption might be that the stimulus-evoked activity sums with the existing natural activity. However, another possibility is that the stimulus-evoked firing of cortical neurons might block and replace the natural activity. We refer to this latter possibility as "neural hijacking." Evidence from analysis of EMG activity evoked by repetitive microstimulation $(200 \mathrm{~Hz}, 500 \mathrm{~ms})$ of primary motor cortex in two rhesus monkeys during performance of a reach-to-grasp task strongly supports the neural hijacking hypothesis.

\section{Introduction}

Repetitive intracortical microstimulation (ICMS) is a popular and highly useful tool for studying the organization and function of cortical motor areas (Asanuma and Rosén, 1972; Andersen et al., 1975; Kwan et al., 1978; Macpherson et al., 1982; Weinrich and Wise, 1982; Lemon et al., 1987; Sato and Tanji, 1989; Schmidt and McIntosh, 1990; Donoghue et al., 1992; Schieber and Deuel, 1997; Baker et al., 1998; Graziano et al., 2002; Schmidlin et al., 2004; Dancause et al., 2006; Burish et al., 2008). It is suprathreshold and capable of evoking movements that can be easily detected as muscle twitches or whole-limb movements. Repetitive ICMS has traditionally been applied as a short-duration train (RSICMS), typically consisting of 10 stimulus pulses at a frequency of $330 \mathrm{~Hz}, 30 \mathrm{~ms}$ duration (Asanuma and Rosén, 1972). This form of repetitive ICMS has been used extensively to map the motor output representation of motor cortex (Asanuma and Rosén, 1972; Andersen et al., 1975; Ethier et al., 2006; Burish et al., 2008). The duration of this form of ICMS produces only brief joint movements and muscle twitches.

To produce stimulus-evoked movements with durations more closely matching natural movements, Graziano et al. (2002) introduced repetitive long-duration ICMS (RL-ICMS) of cortical motor areas. RL-ICMS typically consists of high-frequency trains of stimuli $(200 \mathrm{~Hz})$ lasting $500 \mathrm{~ms}$. An important characteristic of

Received Dec. 5, 2010; revised July 19, 2011; accepted July 25, 2011

Author contributions: D.M.G. and P.D.C. designed research; D.M.G., H.M.H., A.B.-S., and P.D.C. performed research; D.M.G., H.M.H., A.B.-S., and P.D.C. analyzed data; D.M.G., H.M.H., A.B.-S., and P.D.C. wrote the paper.

This work was supported by NIH Grant NS051825 (P.D.C.), NIH Center Grant HD02528 (P.D.C.), and University of Kansas Medical Center Biomedical Research Training Grant (D.M.G.). We thank lan Edwards for technical assistance.

Correspondence should be addressed to Dr. Paul D. Cheney, University of Kansas Medical Center, Department of

Molecular and Integrative Physiology, 3901 Rainbow Boulevard, Mailstop 3043, Kansas City, KS 66160-7336. E-mail: pcheney@kumc.edu.

D. M. Griffin's present address: Systems Neuroscience Institute, University of Pittsburgh School of Medicine, Pittsburgh, PA 15261.

DOI:10.1523/JNEUROSCI.6322-10.2011

Copyright $\odot 2011$ the authors $\quad 0270-6474 / 11 / 3113088-09 \$ 15.00 / 0$
RL-ICMS-evoked movements is their common end-point position regardless of the starting position of the arm (Graziano et al., 2002, 2005). For example, for a particular cortical site, stimulation may produce an arm movement ending with the hand in front of the monkey's torso regardless of the starting position of the hand in the work space surrounding the monkey. Different sites in motor cortex produce different end-point positions of the hand.

Although ICMS methods are used extensively, the mechanism underlying stimulus-evoked muscle activity is not understood in terms of its interaction with natural background activity. One logical possibility is that the stimulus-evoked activity of corticospinal neurons sums with the natural, intrinsic activity. Because corticospinal neurons have direct effects on the activity of motoneurons, changes in their activity will be expressed as changes in EMG activity. Accordingly, if stimulus-evoked cortical activity sums with existing natural activity, then the stimulus-evoked EMG activity would be expected to add to the active movementrelated background activity present at the time stimulation was applied. However, our data demonstrate that this is not the case. Here, we present evidence that ICMS-evoked EMG activity does not sum with the existing background activity; rather, ICMSevoked activity eliminates the background EMG activity and substitutes a new level of EMG activity that is entirely stimulus driven and independent of the existing level of voluntary activity. Our data support a model in which repetitive ICMS blocks natural afferent input to corticospinal neurons and replaces it with stimulus-evoked activity. The results have important implications for the interpretation of experiments in which highfrequency trains of stimulation are applied to cerebral cortex.

\section{Materials and Methods}

Behavioral tasks. RL-ICMS (100 biphasic stimulus pulses at $200 \mathrm{~Hz}, 500$ ms train duration, 60 and $120 \mu \mathrm{A}$ stimulus intensities) was applied to the left M1 of two male rhesus monkeys (Macaca mulatta; $\sim 10 \mathrm{~kg}, 9$ years old) while they performed four behavioral tasks. The tasks were as fol- 
A
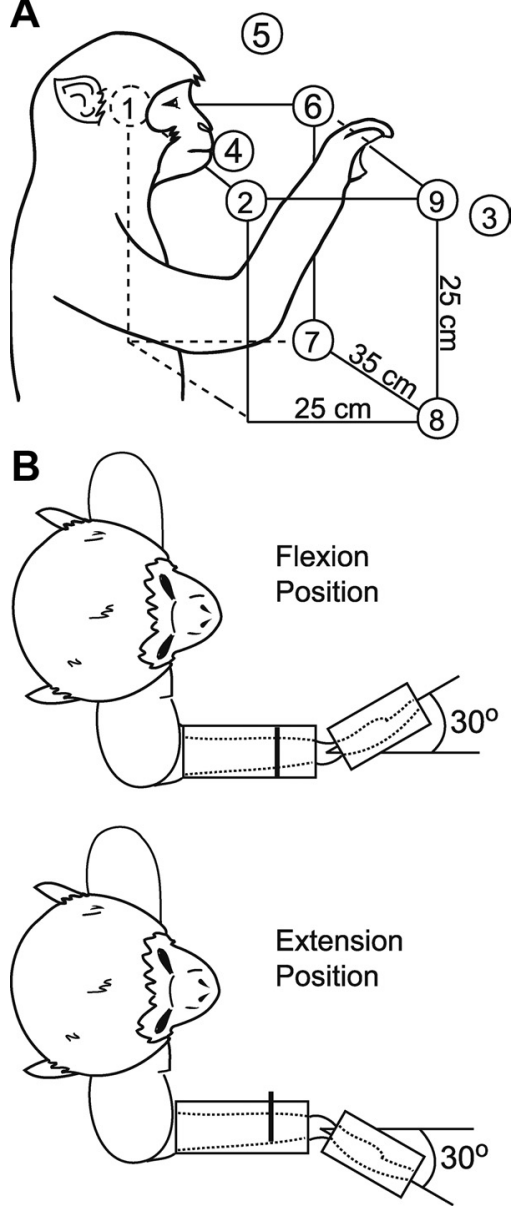

Figure 1. Tasks used to study responses to RL-ICMS. A, Reaching task; circles depict hand starting positions where RL-ICMS was applied. Starting hand positions were achieved either by prompting the monkey to reach for peanuts (circles numbered 1-5) or by rewarding the monkey for grasping a handle attached to a 3-D positioning device (circles numbered 6-9). $\boldsymbol{B}$, Isometric wrist task depicting flexion and extension positions.

lows: (1) reaching with the right hand for a food reward, (2) reaching with the right hand for a handle placed in various positions within the workspace (Fig. 1A), (3) a concentric wrist task in which position alternated between flexion and extension targets, or (4) an isometric wrist task in which the wrist was locked into place at two different positions (Fig. $1 B$ ). During each data collection session, the monkey was seated in a custom-built primate chair inside a sound-attenuating chamber. The left forearm was restrained during task performance. All tasks were performed with the right arm/hand.

Hand starting positions of the reaching tasks are illustrated in Figure $1 \mathrm{~A}$. Peanuts were offered in various positions around the monkey's work space (numbers 1-5; Fig. 1A). RL-ICMS was delivered as the monkey's hand entered the target starting position, but before the monkey grasped the reward. Alternatively, RL-ICMS was delivered as the monkey gripped a handle positioned to serve as an indicator of starting hand position. The handle was locked in place at up to four different positions within the monkey's work space (numbers 6-9; Fig. 1A).

For the wrist tasks (Fig. $1 B$ ), the monkey's lower and upper arm were restrained. The hand, with digits extended, was placed in a padded manipulandum that rotated around the wrist. The wrist was aligned with the axis of rotation of the torque wheel to which the manipulandum was attached. The monkey was required to make self-paced step tracking movements of the wrist alternating between flexion and extension position zones. Both position zones had an inner boundary of $20^{\circ}$ and an outer boundary of $40^{\circ}$. RL-ICMS was delivered at the beginning of the target hold period. For the isometric wrist task, the manipulandum was locked in place at two different wrist positions, including $30^{\circ}$ in flexion and $30^{\circ}$ in extension. The monkey was required to generate ramp and hold trajectories of wrist torque alternately between flexion and extension target zones. The inner and outer boundaries of the torque window were $0.025 \mathrm{~N} \cdot \mathrm{m}$ and $0.05 \mathrm{~N} \cdot \mathrm{m}$, respectively, for flexion and $0.008 \mathrm{~N} \cdot \mathrm{m}$ and $0.025 \mathrm{~N} \cdot \mathrm{m}$, respectively, for extension. RL-ICMS was delivered at the beginning of the target hold period and was limited to once every $3-4$ trials to ensure successful completion of holding within the target zone and delivery of an applesauce reward on a sufficient number of trials to maintain the monkey's interest.

Surgical procedures. After training, a $30 \mathrm{~mm}$ inside diameter titanium chamber was stereotaxically centered over the forelimb area of $\mathrm{M} 1$ on the left hemisphere of each monkey and anchored to the skull with 12 titanium screws (Stryker Leibinger) and dental acrylic (Lux-it). Threaded titanium nuts (Titanium Unlimited) were also attached over the occipital aspect of the skull using 12 additional titanium screws and dental acrylic. These nuts provided a point of attachment for a flexible head restraint system used during data collection sessions. The chambers were centered at anterior $16 \mathrm{~mm}$, lateral $18 \mathrm{~mm}$ (Monkey V) and anterior $16 \mathrm{~mm}$, lateral $22 \mathrm{~mm}$ (Monkey A), at a $30^{\circ}$ angle to the midsagittal plane.

EMG activity was recorded from 24 muscles of the contralateral forelimb with pairs of insulated, multistranded stainless steel wires (Cooner Wire) implanted during an aseptic surgical procedure (Park et al., 2000). Pairs of wires for each muscle were tunneled subcutaneously from an opening above the elbow to their target muscles. The wires of each pair were bared of insulation for $\sim 2-3 \mathrm{~mm}$ at the tip and inserted into the muscle belly with a separation of $\sim 5 \mathrm{~mm}$. Implant locations were confirmed by stimulation through the wire pair and observation of appropriate muscle twitches. EMG connector terminals (ITT Cannon) were affixed to the upper arm using medical adhesive tape. Following surgery, the monkeys wore Kevlar jackets (Lomir Biomedical) reinforced with fine stainless steel mesh (Sperian Protection Americas) to protect the implant. EMG activity was recorded from five shoulder muscles: pectoralis major (PEC), anterior deltoid (ADE), posterior deltoid (PDE), teres major (TMAJ), and latissimus dorsi (LAT); seven elbow muscles: biceps short head (BIS), biceps long head (BIL), brachialis (BRA), brachioradialis (BR), triceps long head (TLON), triceps lateral head (TLAT), and dorsoepitrochlearis (DE); five wrist muscles: extensor carpi radialis (ECR), extensor carpi ulnaris (ECU), flexor carpi radialis (FCR), flexor carpi ulnaris (FCU), and palmaris longus (PL); five digit muscles: extensor digitorum communis (EDC), extensor digitorum 2 and 3 (ED23), extensor digitorum 4 and 5 (ED45), flexor digitorum superficialis (FDS), and flexor digitorum profundus (FDP); and two intrinsic hand muscles: abductor pollicis brevis (APB) and first dorsal interosseus (FDI).

All surgeries were performed under deep general anesthesia and aseptic conditions. Postoperatively, the monkeys were given an analgesic (buprenorphine $0.5 \mathrm{mg} / \mathrm{kg}$ every $12 \mathrm{~h}$ for 3-4 d) and antibiotics (penicillin $\mathrm{G}$, benzathine/procaine combination, $40,000 \mathrm{IU} / \mathrm{kg}$ every $3 \mathrm{~d}$ ). All procedures were in compliance with the guidelines from the Association for Assessment and Accreditation of Laboratory Animal Care (AAALAC) and the Guide for the Care and Use of Laboratory Animals, published by the U.S. Department of Health and Human Services and the National Institutes of Health.

Data collection. Sites in M1 were stimulated using glass and Mylarinsulated platinum-iridium electrodes with impedances ranging from 0.5 to $1.5 \mathrm{M} \Omega$ (Frederick Haer \& Co.). The electrode was positioned within the chamber using an $x-y$-coordinate manipulator and was advanced at approximately a right angle into the cortex with a manual hydraulic microdrive (Frederick Haer \& Co.). Rigid support for the electrode was provided by a 22 gauge guide tube (Small Parts) inside of a 25-mm-long, 3-mm-diameter stainless steel post that touched the surface of the dura.

During electrode penetrations, the first cortical unit activity was noted and the electrode was lowered $1.5 \mathrm{~mm}$ below this point to layer V. Greater depths were required when the electrode track was in the bank of the precentral gyrus. To distinguish layer $\mathrm{V}$ from more superficial layers, particularly in the bank of the precentral gyrus, neuronal activity was evaluated for the presence of large action potentials that were modulated with the task. Stimulus-triggered averages (StTAs) were also collected 
and evaluated for the presence of both clear and robust effects in averages of EMG activity ( $15 \mu \mathrm{A}$ stimulus intensity at $15 \mathrm{~Hz}$ ). Individual stimuli were symmetrical biphasic pulses: a $0.2 \mathrm{~ms}$ negative pulse followed by a $0.2 \mathrm{~ms}$ positive pulse. EMG activity was generally filtered from $30 \mathrm{~Hz}$ to 1 $\mathrm{kHz}$, digitized at a rate of $4 \mathrm{kHz}$, and full-wave rectified.

StTAs were compiled over a $60 \mathrm{~ms}$ epoch, including $20 \mathrm{~ms}$ before the trigger to $40 \mathrm{~ms}$ after the trigger. Mean baseline activity and the SD of baseline EMG activity was measured from the pretrigger period typically consisting of the first $12.5 \mathrm{~ms}$ of each average. StTAs were considered to have a significant poststimulus facilitation (PStF) if the points of the record crossed a level equivalent to $2 \mathrm{SD}$ of the mean of the baseline EMG for a period $\geq 0.75 \mathrm{~ms}$ ( 3 points) or more (Park et al., 2001). Note that an effect with a width of $0.75 \mathrm{~ms}$ at the peak would typically have a much longer duration, in the range of 3-4 ms, at its base. StTAs with clear and robust effects typically had PStF peaks $>4$ SD of baseline mean activity.

Assessment of stimulus-triggered averages. StTAs were collected $(15 \mu \mathrm{A}$ stimulus intensity at $15 \mathrm{~Hz}$, symmetrical biphasic pulses) at identified layer V sites in forelimb M1. The assessment of StTA effects was based on averages of at least 500 trigger events. Segments of EMG activity associated with each stimulus were evaluated and accepted for averaging only when the mean of all EMG data points over the entire $60 \mathrm{~ms}$ epoch was $\geq 5 \%$ of full-scale input. This prevented averaging segments in which EMG activity was minimal or absent (McKiernan et al., 1998). EMG recordings were tested for cross talk by computing EMG-triggered averages (Cheney and Fetz, 1980). This procedure involved using the EMG peaks from one muscle as triggers for compiling averages of rectified EMG activity from that muscle and all other muscles. Most muscles showed no evidence of cross talk. However, in muscles that did have cross-talk peaks, we still accepted the effect as valid if the ratio of poststimulus facilitation (PStF) between the test and trigger muscles exceeded the ratio of their cross-talk peaks by a factor of two or more (Buys et al., 1986). Based on this criterion, none of the effects obtained in this study were eliminated.

RL-ICMS-evoked EMG activity. Layer V sites with clear and robust StTA effects in forelimb muscles were identified and selected for data collection with RL-ICMS. RL-ICMS consisted of a train of 100 symmetrical biphasic stimulus pulses at $200 \mathrm{~Hz}$ (500 ms train) using either 60 or $120 \mu \mathrm{A}$ intensity. Although high relative to threshold for twitch responses, intensities in this range were necessary to produce complete movements to consistent end-points. It should also be noted that these intensities did not produce a "ceiling effect" in EMG activity because further increases in intensity produced further increases in the level of EMG activity.

The assessment of effects was based on averages of $4-8$ stimulus trains. Averages of RL-ICMS-evoked EMG activity were compiled over a $1.2 \mathrm{~s}$ epoch, including $200 \mathrm{~ms}$ before the trigger to $1000 \mathrm{~ms}$ after the trigger. Mean baseline activity was measured from the pretrigger period typically consisting of the first $100 \mathrm{~ms}$ of each average. The first pulse of each train was used as a trigger to compute averages of RL-ICMS-evoked EMG activity. The magnitude of the EMG response was expressed as the mean EMG level present from the onset to the termination of the response identified as the points where the record crossed a level equal to 2 SD of the baseline points.

Imaging. Structural MRIs were obtained from a 3 tesla Siemens Allegra system. Images were obtained with the monkey's head mounted in an MRI compatible stereotaxic apparatus so the orientation and location of the cortical recording chamber and electrode track penetrations could be determined. Two-dimensional renderings of experimental sites were constructed for each monkey. The method for flattening and unfolding cortical layer $\mathrm{V}$ in the anterior bank of the central sulcus has been previously described in detail (Park et al., 2001). Briefly, the cortex was unfolded and the locations of experimental sites were mapped onto a two-dimensional cortical sheet based on the electrode's depth and $x-y$-coordinate, known architectural landmarks, MRI images and observations noted during the cortical implant surgeries.

\section{Results}

We obtained data from the left M1 cortex in two rhesus monkeys. RL-ICMS (100 biphasic stimulus pulses at $200 \mathrm{~Hz}, 500 \mathrm{~ms}$ train duration)-triggered averages of EMG activity were collected at a total of 42 sites while the monkeys performed a whole-limb reaching task or an isolated wrist movement task (Fig. 1). We used stimulus intensities that produced consistent hand endpoint positions around the monkey's workspace (60 and 120 $\mu \mathrm{A})$. The data included 14 sites in monkey $\mathrm{V}$ and 28 sites in monkey A. A total of 2736 RL-ICMS-triggered averages of EMG activity were analyzed yielding 1615 averages in which RL-ICMS had a significant effect on EMG activity. Most of these produced an increase in the existing level of EMG activity regardless of the initial active movement conditions (starting hand position). However, $5 \%$ of effects $(82 / 1615)$ were instances in which RLICMS applied to the same cortical site appeared to produce opposite effects (suppression or excitation) depending on the initial level of EMG activity. At starting hand positions where background EMG level was high, RL-ICMS reduced EMG activity. While at other positions, where background EMG activity was low, RL-ICMS increased EMG activity. Although data from all sites are relevant, sites where ICMS produced opposite effects depending on the prestimulus levels of EMG activity were particularly powerful in revealing a fundamental characteristic of ICMS-evoked cortical activation.

RL-ICMS appears to produce opposing muscle responses (suppression in one case and excitation in the other) depending on the prestimulus level of voluntary EMG activity (records 1-4; Fig. 2). The monkey illustrations at the top of each panel show the starting hand positions (also see Fig. 1) used to produce different levels of background EMG activity. The examples were derived from two cortical sites (50A1 - upper panel, 41A1-lower panel) and three muscles (LAT, DE, TLON). Column A illustrates the condition in which RL-ICMS (shaded area) produced increases in EMG activity from a relatively low initial prestimulus baseline level. Column B illustrates the condition in which RL-ICMS produced decreases in EMG activity from a relatively high initial prestimulus baseline level. Column $\mathrm{C}$ contains superimposed EMG records from columns A and B. At the cortical site tested in the upper panel, RL-ICMS consistently drove the hand to a final end-point position near the monkey's abdomen regardless of the starting position. In this example, RL-ICMS produced small but consistent increases in EMG activity of LAT and DE when the hand was near the mouth (column A) and background (prestimulus) EMG activity was low. In contrast, when RL-ICMS was applied with the hand centered near the waist and background EMG activity was high, stimulation produced what appears to be a profound suppression of EMG activity. Clearly, stimulusevoked activity is not summing with the ongoing natural movement-related activity. Rather, the data suggest that a process of elimination and substitution is occurring. The large increase in activity shortly following termination of the stimulus is resumption of voluntary EMG activity. The lower example shows similar results for another cortical site and two muscles. At this site, RL-ICMS drove the hand to a final end-point position near the monkey's chest. RL-ICMS produced an increase in EMG activity from baseline when the hand started at position 8 (column A) and a decrease in activity when the hand started at position 7 (column B). However, in both examples, the overall mean level of stimulus-driven EMG activation was very similar regardless of starting hand position. For the cortical site in the upper panel, the mean levels of EMG activity during stimulation at the two starting hand positions differed by $9.7 \%$ for LAT and only $4 \%$ for DE. For the lower panel, the mean differences were $26 \%$ for TLON and $16 \%$ for DE. However, it is important to note that by the end of the stimulus train, the two EMG records show identical levels 

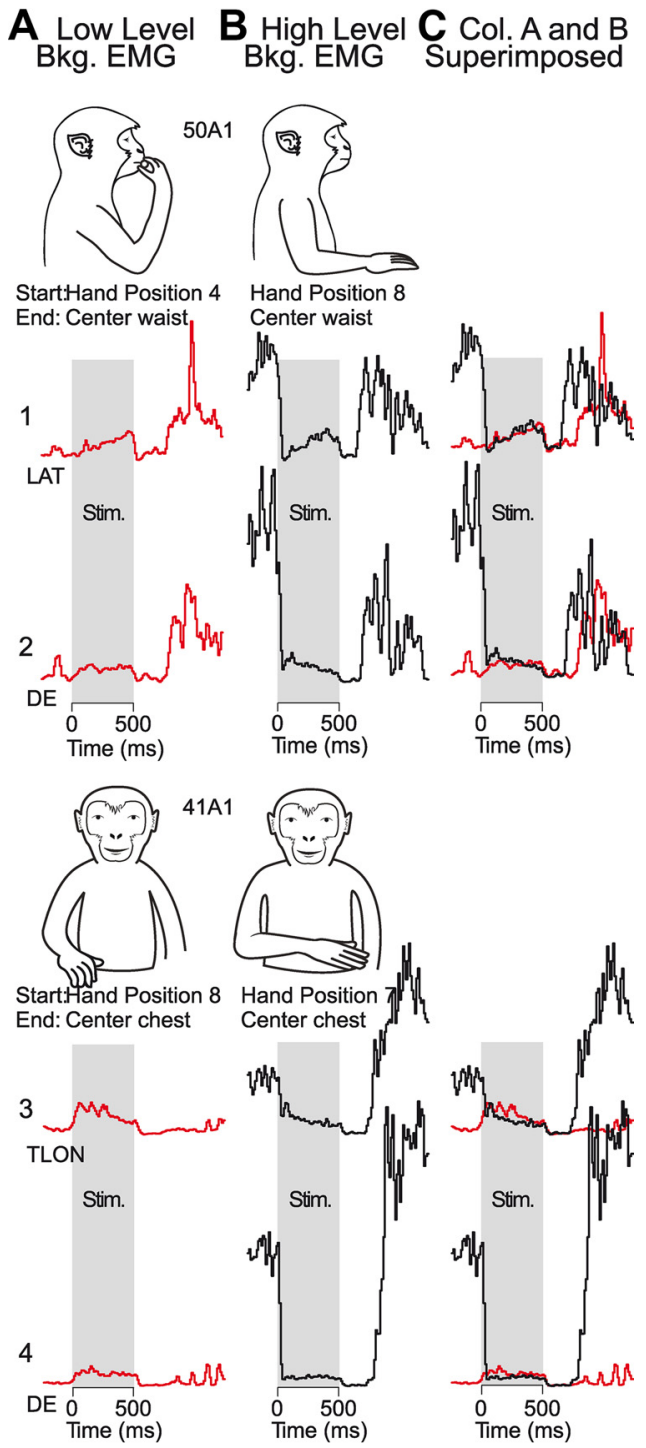

Figure 2. Examples illustrating "hijacking" of cortical output by high-frequency $(200 \mathrm{~Hz})$, long-duration (500 ms) repetitive stimulation (RL-ICMS) at two cortical sites (upper panel: 120 $\mu \mathrm{A}$ and lower panel: $60 \mu \mathrm{A}$ ). Gray shading represents the duration of the stimulus (Stim.) train (500 ms). A, RL-ICMS-evoked EMG activity when stimulation was applied in the presence of a low level of prestimulus background (bkg.) EMG activity. B, RL-ICMS-evoked EMG activity when stimulation was applied in the presence of a high level of prestimulus background EMG activity. C, Superimposition of EMG records from Columns (Col.) $\boldsymbol{A}$ and $\boldsymbol{B}$. See Figure 1 for starting hand positions. Corresponding records in columns $\boldsymbol{A}$ and $\boldsymbol{B}$ are displayed at the same scale.

of activity. In fact, it is striking how similar the basic patterns of stimulus-driven EMG activity are at the two different starting positions, despite the fact that in one case the stimulus-evoked activity falls from a high preexisting EMG level while in the other case it rises from a low preexisting EMG level. This is evident in column $\mathrm{C}$, in which the EMG records corresponding to the two starting positions are superimposed. For example, LAT (upper panel) shows a ramp increase pattern during the stimulus train in both EMG records. TLON (lower panel) shows a ramp decrease pattern during the stimulus train in both EMG records. In the records for DE (lower panel), the RL-ICMS-evoked activity pattern remains tonic throughout the stimulus train. Most importantly, in all cases the records are virtually superimposable, particularly near the end of the stimulus train.

Based on the data in Figure 2, the effect of stimulation from the same cortical site appears to be excitation at one hand posi-

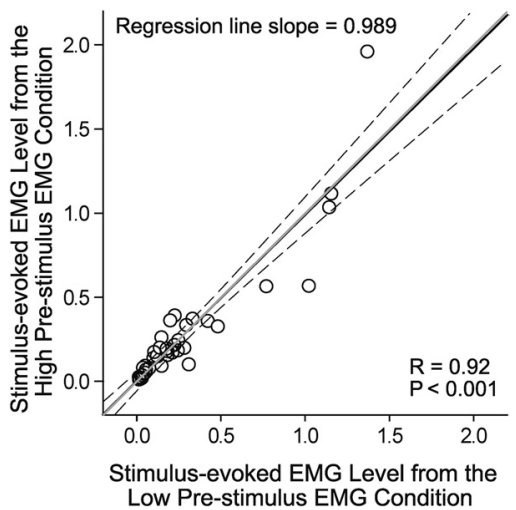

Figure 3. Relationship between RL-ICMS-evoked mean EMG levels at two different starting hand positions for 41 cortical site-muscle pairs that produced opposing effects on EMG activity. The black line is the linear regression line. Dotted lines are $95 \%$ confidence intervals. The gray line has a slope $=1$. The regression line slope, correlation coefficient $(R)$, and $p$ value are given. EMG activity is in arbitrary units.

tion and suppression at another hand position. However, in both cases the same level of EMG activity was achieved during stimulation, suggesting that the stimulus-evoked activity did not switch between excitation and suppression depending on the starting position of the hand, but rather that high-frequency stimulation eliminated the natural movement-related activity of corticospinal neurons and substituted activity that was solely stimulus evoked. We refer to this as "hijacking" of cortical output.

We further plotted RL-ICMS-evoked EMG activity level at one starting hand position against the EMG activity level at the second hand position for all 41 cortical site-muscle pairs that produced opposing qualitative effects (Fig. 3). The scatter plot has a correlation coefficient of $0.92(p<0.001)$ and a regression slope of 0.99 , demonstrating that RL-ICMS evoked nearly the same level of EMG activity regardless of the starting hand position or the prestimulus level of EMG activity. RL-ICMS forced a new level of EMG activity that was independent of background EMG activity.

Cortical site-muscle pairs in which stimulation evoked an intermediate level of EMG activity, between lower and higher voluntary levels (Fig. 2), provide the most compelling evidence for RL-ICMS hijacking of cortical motor output. Although there were only 41 of these cortical site-muscle pairs (Fig. 3), all 557 additional site-muscle pairs demonstrate the same principle. As with opposite stimulus-evoked responses, stimulation at these additional sites evoked a level of EMG activity that did not sum with the prestimulus active movement-related level of background EMG. Rather, stimulation produced the same level of EMG activity regardless of the prestimulus level, even when there was greater than a $100 \%$ difference between the two prestimulus activity levels (Fig. 4). The data show effects of RL-ICMS on forearm extensor (blue traces) and flexor (red traces) muscles at one cortical site when stimulation was applied with the starting position of the wrist in extension (Fig. $4 A$ ) and flexion (Fig. $4 B$ ). Stimulation was applied during the period indicated by gray shading. The black record is wrist position, which reflects a combination of voluntary and RL-ICMS-generated forces. The wrist position record shows a transient movement toward flexion after the start of the stimulus train (Fig. 4A), which we attribute to a dominant initial burst of flexor muscle activity while the wrist was extended. However, for both starting wrist positions $\left(30^{\circ}\right.$ extension and $30^{\circ}$ flexion), RL-ICMS either extended the wrist (Fig. $4 B$ ) or maintained the wrist in extension (Fig. $4 A$ ). As ex- 


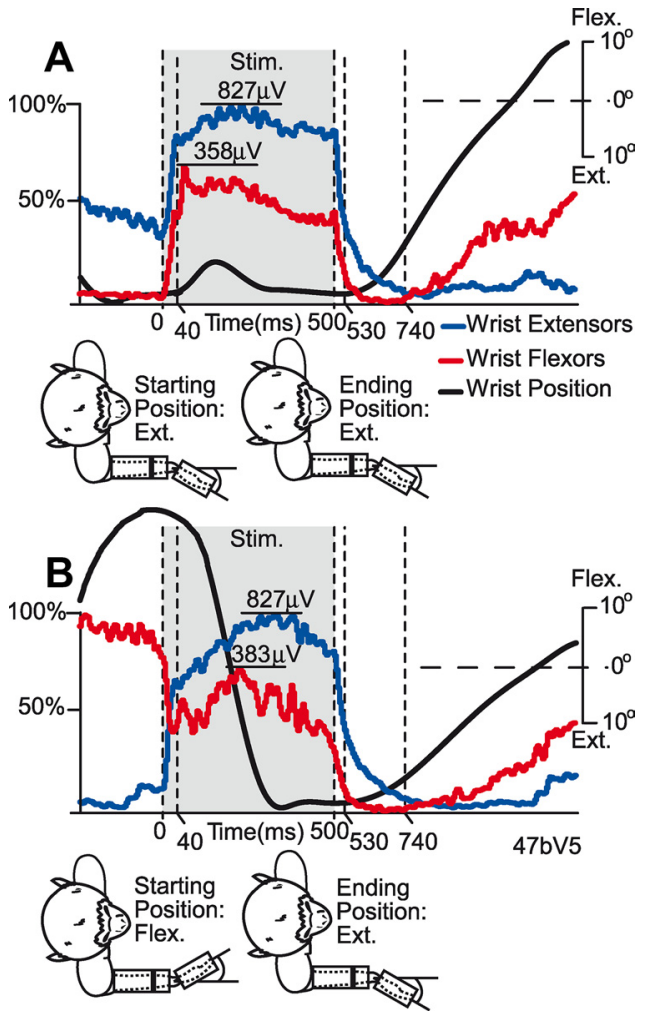

Figure 4. RL-ICMS-evoked EMG levels in wrist muscles. Extensor (blue traces) and flexor (red traces) muscle activity at a single cortical site obtained while the monkey performed a concentric wrist movement task. The monkey drawings below each set of records show the position of the wrist before RL-ICMS and at its termination. RL-ICMS was applied with wrist extended (ext.) (A) and flexed (flex.) (B). Gray shading represents the 500 ms period where RL-ICMS (Stim.) was applied. Wrist extensor EMG record is the sum of EDC, ED23, ED45, ECR, and ECU; the flexor EMG record is the sum of FDS, FDP, FCR, FCU, and PL. The black trace is wrist position. EMG amplitude is quantified as the percentage of maximum observed within each average record. However, to facilitate comparison of EMG levels obtained under extension versus flexion, the absolute level of EMG activity in microvolts is also given for the peak activity during stimulation.

pected, prestimulus wrist extensor muscle activity was relatively high when the monkey was actively holding the wrist in an extended position (blue record; Fig. $4 \mathrm{~A}$ ) and low when holding in a flexed position (blue record; Fig. $4 B$ ). Most importantly, RLICMS drove extensor muscle activity to the same absolute level during stimulation $(827 \mu \mathrm{V})$ regardless of position-related differences in the prestimulus level of activity (compare the peaks of the blue records in Fig. $4 A, B$ ). The increase from baseline with the wrist in extension was $532 \mu \mathrm{V}$ (Fig. 4A) compared to $774 \mu \mathrm{V}$ with the wrist in flexion (Fig. $4 B$ ). If stimulus-evoked activity had simply summed with existing voluntary EMG activity, the EMG level attained during stimulation should have been much larger when prestimulus EMG was high (Fig. $4 A$ ) than when it was low (Fig. $4 B$ ); but instead the final levels were nearly the same. The same result was obtained for each of the individual extensor muscles that were summed together to yield the blue records (Fig. 4). These results demonstrate that the phenomenon of replacement (hijacking) is a consistent feature of cortical activation with highfrequency ICMS and is not limited to sites where ICMS produces opposing responses depending on the prestimulus baseline activity.

Flexor muscle activity (red trace; Fig. 4) responded oppositely to stimulation relative to the prestimulus EMG level. This is another example of the type of response illustrated previously (Fig. 2 ). When the wrist was in extension (Fig. 4A) and flexor EMG

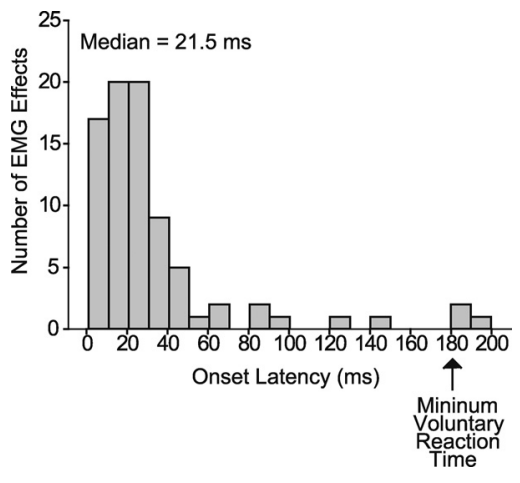

Figure 5. Distribution of stimulus-evoked EMG onset latencies. Onset latency was measured relative to the stimulus train onset. Minimum voluntary reaction time to a somatosensory stimulus is given as $180 \mathrm{~ms}$ (Nelson et al., 1990; Naito et al., 2000).

activity was low, stimulation produced a large increase in activity. In contrast, when the wrist was in flexion and flexor activity was high, stimulation decreased activity (Fig. 4B). However, as with previous examples (Fig. 2), the stimulus-driven level of activity was very similar regardless of the starting conditions $(358 \mu \mathrm{V}$ in Fig. $4 A$ vs $383 \mu \mathrm{V}$ in Fig. $4 B$ ). The stimulus-evoked level of activity did not sum with prestimulus voluntary activity. Rather, a new level of activity was attained during stimulation that was independent of prestimulus conditions.

Could the responses to stimulation include a voluntary reaction to the stimulus? For instance, could the decrease in EMG activity be due to the monkey "letting go" from the sensation of the motor effects of the stimulus, and could the increase in EMG activity be related to the monkey voluntarily increasing EMG activity to oppose the effects of the stimulus (hand moving away from the target)? Figure 5 is a summary of the stimulus-evoked EMG activation onset latencies for all 41 cortical site-muscle pairs in which stimulation produced opposing effects depending on the prestimulus EMG level. Ninety-six percent of the latencies are less than the expected minimum reaction time to a somatosensory stimulus (180 ms) (Nelson et al., 1990; Naito et al., 2000), suggesting that changes in voluntary effort do not contribute to the initial phase of the EMG response to stimulation and this is not a viable alternative explanation to hijacking.

Events associated with the termination of the stimulus train are also of interest. Is the voluntary active movement signal still present when stimulation ends? The data show that, in fact, the voluntary EMG signal (present before the stimulus train was applied) is not present at stimulus termination (Fig. 4). If it were, the wrist extensor record should return to the level of EMG activity present before the onset of stimulation $(\sim 295 \mu \mathrm{V}$; Fig. $4 A)$ and the wrist flexors should rise to $\sim 466 \mu \mathrm{V}$ (Fig. $4 B$ ). However, these prestimulus EMG levels were not achieved. Instead, at the end of the stimulus train, both flexor and extensor muscle activity drops to near zero over a period of $240 \mathrm{~ms}$, suggesting that at some point during the stimulus train, the internal motor program for voluntary movement was terminated. It is also important to note that aside from the decrease in EMG level at stimulus onset, there were no decreases during stimulation that would reflect an abrupt termination of voluntary effort. This result further suggests that cortical activity related to voluntary effort is being blocked by the stimulus beginning at stimulus onset and continuing throughout the stimulus train. Because voluntary effort-related activity is essentially masked by the effects of the stimulus train, there is no change in EMG activity reflecting the 
termination of voluntary effort that must have occurred somewhere during the stimulus train.

Voluntary responses were reinitiated $\sim 300 \mathrm{~ms}$ after stimulus termination (Fig. 4). In one case (Fig. 4B), the new voluntary response is in the same direction as the prestimulus response (flexion) because a reward was not obtained on this trial. In the other case (Fig. 4A), the new voluntary response (flexion) was opposite the prestimulus response because the monkey did receive a reward for the extension trial. In both cases, a spring-like load centered at zero position assists the new voluntary response in moving the manipulandum in the flexion direction.

RL-ICMS typically hijacks cortical output within the first 50 ms of the stimulus train (Fig. 5). At that time, the limb starts to move in response to the new levels of muscle activity (as evident in the wrist position trace of Fig. 4). Is it possible that the spinal cord interneuronal circuitry translates the descending, stimulusdriven signal and modifies motoneuronal activity to produce different directions of movement necessary to achieve the same final common hand position? The interneuronal circuitry of the spinal cord could potentially modify the input to motoneurons based on changing afferent input associated with different static limb positions and with dynamically changing positions associated with stimulus-evoked movement. If that were the case, one might expect to see higher variability in EMG activity at the beginning of the stimulus-evoked response because the initial limb position is variable. On the other hand, one would expect to see lower variability at the end of the stimulus-evoked effect, because the limb has achieved its final common end-point position. We investigated this possibility using two measures. First, we compared the $\mathrm{SD}$ of the first and last $100 \mathrm{~ms}$ of stimulus-driven EMG activity across all the starting hand positions for 23 cortical site-muscle pairs where it was possible to test four or more starting hand positions with RL-ICMS. The first $100 \mathrm{~ms}$ of the stimulus-driven EMG record was measured starting with a point in time when activity stabilized after a brief transition period ( $\sim 20-40 \mathrm{~ms})$ associated with the stimulus onset. This was not an issue with stimulus termination because EMG changes related to termination were delayed from the end of stimulation. The SDs for the first and last $100 \mathrm{~ms}$ of the EMG records could be derived for each of the 23 sites because each site was tested with four or more hand positions. The median SD for the dataset representing the first $100 \mathrm{~ms}$ of stimulus-evoked EMG activity measured across all 23 sites was 0.0998 . The median SD for the final $100 \mathrm{~ms}$ of stimulusevoked muscle activity was $42 \%$ lower (0.0578), although this difference did not achieve statistical significance $(p=0.15$, Wilcoxon signed rank test). We also examined this issue by calculating the variability in stimulus-evoked EMG responses for the first and last $100 \mathrm{~ms}$ as a percentage of the mean EMG during each period. The median variability in EMG responses for the first 100 ms expressed as a percentage of the mean was 35\% compared to $23 \%$ for the last $100 \mathrm{~ms}$ of stimulation. This difference was statistically significant ( $p<0.01$, Wilcoxon signed rank test). The lower level of variability in EMG responses at the end of stimulation compared to the beginning may reflect the actions of afferent input not only on motoneurons directly but also on the spinal cord interneuronal network. Afferent input should be less variable at the end of movement because limb position was less variable than at the onset of movement.

Much of our analysis so far has focused on cortical site-muscle pairs where RL-ICMS evoked a decrease from baseline EMG at one starting position and an increase from baseline at another starting position. Although these were instances where the monkey was producing highly variable voluntary muscle activity as-

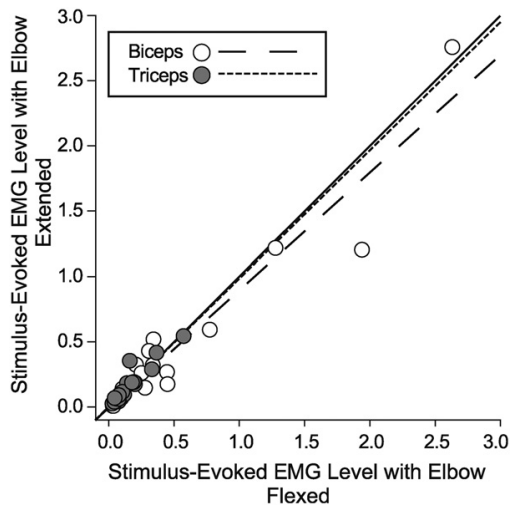

Figure 6. Relationship between RL-ICMS-evoked mean EMG levels at two starting hand positions associated with extremes of elbow flexion and extension. The solid line has a slope $=$ 1. Gray dots represent elbow extensors (triceps) and white dots represent elbow flexors (biceps). EMG activity is in arbitrary units.

sociated with each starting hand position, these positions may not have been those associated with the largest changes in joint angle for each analyzed muscle. Does the hijacking principle of consistent muscle activation independent of initial limb position remain intact if positions associated with large changes in joint angle are compared? For this analysis, we chose the elbow joint because of the extremes of joint angle available in our dataset. We then tested two starting hand positions that produced extremes of elbow flexion (hand position 4 in Fig. 1) and elbow extension (hand position 3 in Fig. 1). The cortical sites chosen were all ones in which RL-ICMS drove the hand to a final position in front of the monkey. We measured RL-ICMS-evoked effects from biceps (BIS, BIL) and triceps (TLAT, TLON). We plotted mean RLICMS-evoked EMG activity levels in elbow muscles with the elbow flexed against EMG levels with the elbow extended (Fig. 6). As with other cases presented previously, RL-ICMS evoked similar levels of activity at both extremes of elbow position. The regression lines for both elbow flexors and extensors are very close to the unity line (biceps regression slope $=0.90$, triceps regression slope $=0.98$ ). The regression line for the biceps muscles is slightly shifted to the right of unity, which reflects a few instances where RL-ICMS evoked a higher level of activation when the elbow was flexed.

\section{Discussion}

In this study, 41 cortical site-muscle pairs produced opposing RL-ICMS-evoked EMG responses (increase in one case, decrease in the other) depending on task conditions and the associated prestimulus level of EMG activity. These opposing responses give the appearance of excitation in one condition and suppression in another condition. In other words, the output sign appears to change based on limb posture or joint position (Graziano et al., 2004). An alternative explanation, strongly supported by our results, is that high-frequency repetitive stimulation takes over (hijacks) cortical output by blocking the natural voluntary movement-related activity and replaces it with activity that is driven solely by stimulation, independent of existing behavioral conditions. This interpretation is supported by the fact that at all sites tested, RL-ICMS drove EMG activity to the same or nearly the same level regardless of the initial conditions, including positions representing the extremes of joint angle. If RL-ICMS evoked excitation at one limb posture/joint position and suppression at another, it is highly improbable that the level of EMG activity achieved under each condition 


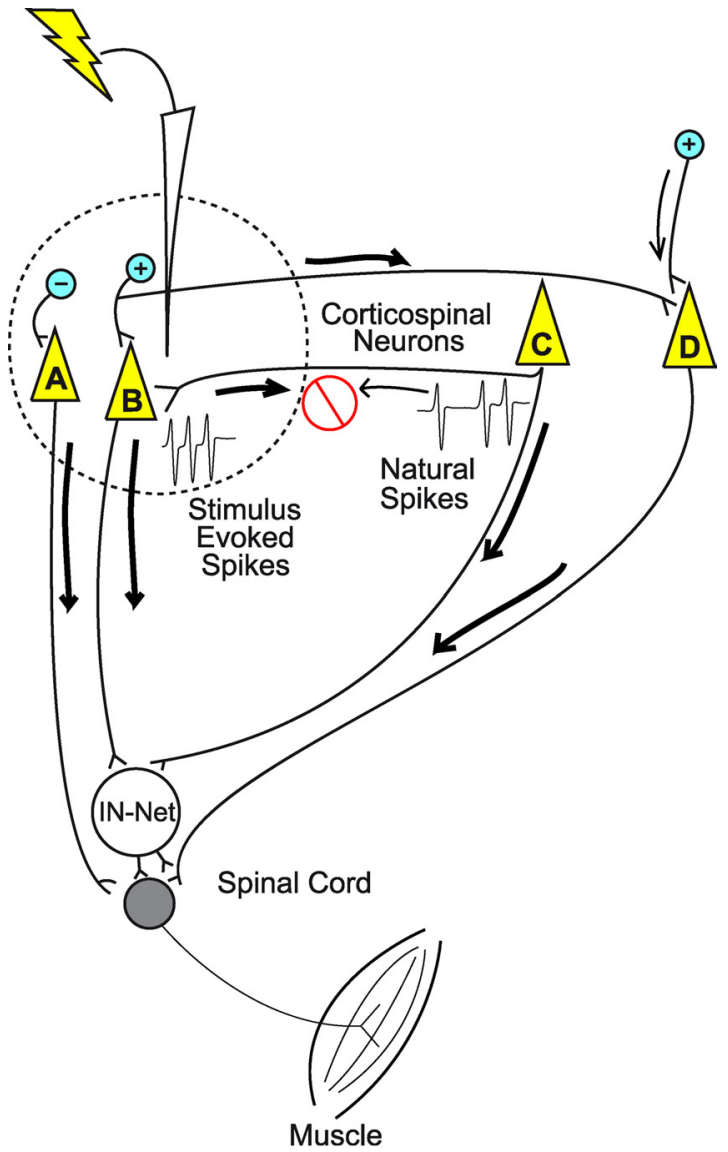

Figure 7. Illustration of proposed cortical "hijacking" mechanism of RL-ICMS evoked EMG activation. The dotted line represents the physical spread of current from the stimulating microelectrode. Corticospinal neurons $A$ and $B$ are present within the sphere of activation along with GABA inhibitory interneurons $(-)$ and excitatory interneurons $(+)$. Stimulus-evoked spikes (heavy arrows) travel orthodromically along descending axons and both orthodromically and antidromically along horizontal axon collaterals. Antidromic spikes collide with and block naturally occurring orthodromic spikes (light arrows) resulting in complete replacement of natural spikes with stimulus-evoked spikes depending on the stimulus frequency. The cell bodies of corticospinal neurons $C$ and $D$ are outside the area of direct activation. One cortical neuron's axon (D) is not activated antidromically by the stimulus, but it does receive stimulus-driven orthodromic input. Stimulus-driven corticospinal output influences motoneurons directly and also through the spinal cord interneuronal network (IN-Net).

would be the same, especially for all 41 cortical sites that yielded opposing responses.

Although this study focused on the 41 cortical site-muscle pairs that produced opposing responses ( $6 \%$ of total), the same basic result also applies to the other $94 \%$ of cortical site-muscle pairs studied. For example, for cortical site-muscle pairs that showed only increases in stimulus-evoked activity, the final level of EMG activity achieved was the same regardless of the initial conditions, including the initial level of EMG activity. Therefore, it appears that the lack of summation at the level of motoneuron activity between stimulus-evoked activity and natural voluntary activity is the rule rather than the exception for all cortical sites where high-frequency stimulation was applied.

Our findings suggest that high-frequency ICMS hijacks natural cortical activity and replaces it with stimulus-evoked activity. Possible mechanisms are illustrated in Figure 7. Four corticospinal neurons (A-D) are represented. The sphere defined by the dotted line represents the cortical volume containing neural elements directly activated by the stimulus. Neurons within the sphere of activation are most likely activated by two mechanisms (Stoney et al., 1968; Ranck, 1975; Asanuma et al., 1976; Marcus et al., 1979): (1) direct excitation at the cell's initial segment, an axon collateral, or the cell body itself, and (2) indirect, transsynaptic excitation from stimulated afferent axons. In neurons directly activated by the stimulus (A and $B$ ), regardless of the mechanism, spikes evoked will propagate orthodromically down the axon and antidromically back into the cell body. If the stimulus intensity is suprathreshold, the neuron will be depolarized to firing threshold with every stimulus regardless of where the stimulus occurs relative to naturally occurring spikes (other than the absolute refractory period). We propose that replacement of the natural activity of corticospinal output neurons with stimulusdriven activity (hijacking) occurs when the frequency of stimulation exceeds the frequency of naturally occurring spikes.

Stimulation of axon terminals will give rise to both orthodromic (Hashimoto et al., 2003) and antidromic (Li et al., 2007) spikes. Antidromic spikes in axon collaterals will propagate back to branch points and then conduct orthodromically to targets (neuron C in Fig. 7). This could result in direct activation of a neuron even though the cell body lies some distance from the site of stimulation (Histed et al., 2009). But once again, if the frequency of stimulus-evoked spikes exceeds the frequency of naturally occurring spikes, all of the naturally occurring spikes on the afferent axon (light arrow) will be blocked by collision with stimulus-evoked spikes (heavy arrow). Complete replacement of voluntary EMG activity in a muscle with stimulus-driven activity will occur when the stimulus intensity is sufficiently high that all the corticospinal output neurons mediating a muscle's natural activity have been hijacked. Accordingly, effective hijacking requires a minimum combination of stimulus frequency and intensity.

Another factor that may contribute to the elimination of ongoing natural cortical activity is activation of cortical inhibitory interneurons (GABA) and synaptic terminations (Fig. 7). The presence of GABA neurons in motor cortex is well established (Hendry and Jones, 1981). Moreover, GABA can exert a potent inhibitory action on motor cortex neurons, in some cases producing complete suppression of movement-related activity (Matsumura et al., 1992).

The hijacking mechanism described above leads to some additional interesting issues in the context of Figure 7. First, complete replacement of natural activity was observed at relatively high stimulus intensities $(60-120 \mu \mathrm{A})$ when delivered at frequencies of $200 \mathrm{~Hz}$. With these parameters, the monkey seemed unable to overcome the effects of stimulation and behavioral performance was completely interrupted. Using even a minimal value of $k$ in the expression $r=\sqrt{i / k}$, where $r$ is the radius of effective activation of neuronal elements and $i$ is the stimulus current, at $120 \mu \mathrm{A}$ the expected physical spread of excitatory current would be a sphere of radius $0.69 \mathrm{~mm}$, which yields a cortical surface area of $1.5 \mathrm{~mm}^{2}$ (Cheney and Fetz, 1985). In comparison, the area of cortical representation for typical hand/ digit muscles is $15-20 \mathrm{~mm}^{2}$ (Andersen et al., 1975; Park, 2002). In view of this, how does RL-ICMS activation of neural elements within a sphere of radius $0.69 \mathrm{~mm}$ hijack all the cortical neurons that supply a particular motoneuron pool? One possibility is based on the fact that corticospinal neurons with the same target muscles are highly interconnected through axon collaterals and branching afferent inputs (Jackson et al., 2003; Smith and Fetz, 2009a,b). As a result, even corticospinal neurons located some distance from the site of stimulation could be hijacked and the cortical area affected by stimulation could expand well beyond the site of stimulation. Tolias et al. (2005) used fMRI to measure the area of activation of visual cortex with microstimulation and concluded that the activated area includes both a sphere of direct 
excitation and a broader region activated transsynaptically. At current levels of $159-1651 \mu \mathrm{A}(100 \mathrm{~Hz}$ for $4 \mathrm{~s})$, they reported activation up to $4.5 \mathrm{~mm}$ from the electrode tip. Assuming a circular area of activation, this corresponds to a cortical area of 64 $\mathrm{mm}^{2}$, which in M1 cortex could encompass the entire representation of a digit muscle. Of course, the currents they used were generally considerably greater than those applied in this study. However, Seidemann et al. (2002), using optical imaging, reported activation with microstimulation $(50 \mu \mathrm{A}, 500 \mathrm{~Hz}, 30 \mathrm{~ms}$ train) of an area $\sim 4.5 \times 3.5 \mathrm{~mm}$ centered around a microelectrode in the frontal eye field. Slovin et al. (2003), also using optical imaging methods in M1 cortex of awake monkeys, found that single microstimuli ranging from 15 to $30 \mu \mathrm{A}$ could produce 1.5to 3 -mm-wide areas of activation. In layer $2 / 3$ of cat visual cortex, Histed et al. (2009) found neuronal activation up to $4 \mathrm{~mm}$ away from the stimulating electrode with currents as low as $10 \mu \mathrm{A}$. These relatively large areas of activation might occur by transmission over axon collaterals that have been shown to extend over relatively large distances in the cortex from the cell bodies of origin. Using both retrograde and anterograde tracer methods in motor cortex, labeling at distances up to $7-8 \mathrm{~mm}$ from the site of injection has been reported, although bouton density was greatest within $1.0-1.5 \mathrm{~mm}$ of the injection site and decreased progressively with distance from the injection site (Huntley and Jones, 1991; Keller, 1993; Keller and Asanuma, 1993; Capaday et al., 2009). The concentration of intracortical connections within a radius of $1.5 \mathrm{~mm}$ from a particular point is also consistent with electrophysiological studies of synaptic interactions between neurons revealed with cross-correlation methods (Hatsopoulos et al., 1998; Jackson et al., 2003; Smith and Fetz, 2009a,b). Corticomotoneuronal cells with common target muscles show the strongest synaptic interactions (Jackson et al., 2003; Smith and Fetz, 2009a). Taking all of these findings into account and given the stimulus parameters that we used, it seems possible that RLICMS trains could have affected, either directly and/or transsynaptically, not only the entire representation of an individual forelimb muscle but potentially the entire $\mathrm{M} 1$ forelimb representation (Park et al., 2001).

Finally, it should be noted that the hijacking mechanism proposed here for microstimulation in the cortex is similar to the mechanism described by Garcia et al. $(2003,2005)$ to explain the action of high-frequency stimulation $(80-185 \mathrm{~Hz})$ of the subthalamic nucleus used to treat Parkinson's disease. They found that high-frequency stimulation replaced the preexisting spontaneous neuronal activity with spikes that were time locked to individual stimulus pulses.

To conclude, our results suggest that high-frequency ICMS blocks naturally occurring spikes generated by the internal motor program for the activation of corticospinal output neurons. These natural signals are then replaced with output signals that are driven solely by the applied stimulus train. In this sense, highfrequency ICMS can be viewed as "hijacking" cortical output to motoneurons.

\section{References}

Andersen P, Hagan PJ, Phillips CG, Powell TP (1975) Mapping by microstimulation of overlapping projections from area 4 to motor units of the baboon's hand. Proc R Soc Lond B Biol Sci 188:31-36.

Asanuma H, Rosén I (1972) Topographical organization of cortical efferent zones projecting to distal forelimb muscles in the monkey. Exp Brain Res 14:243-256.

Asanuma H, Arnold A, Zarzecki P (1976) Further study on the excitation of pyramidal tract cells by intracortical microstimulation. Exp Brain Res 26:443-461.
Baker SN, Olivier E, Lemon RN (1998) An investigation of the intrinsic circuitry of the motor cortex of the monkey using intra-cortical microstimulation. Exp Brain Res 123:397-411.

Burish MJ, Stepniewska I, Kaas JH (2008) Microstimulation and architectonics of frontoparietal cortex in common marmosets (Callithrix jacchus). J Comp Neurol 507:1151-1168.

Buys EJ, Lemon RN, Mantel GW, Muir RB (1986) Selective facilitation of different hand muscles by single corticospinal neurones in the conscious monkey. J Physiol 381:529-549.

Capaday C, Ethier C, Brizzi L, Sik A, van Vreeswijk C, Gingras D (2009) On the nature of the intrinsic connectivity of the cat motor cortex: evidence for a recurrent neural network topology. J Neurophysiol 102:2131-2141.

Cheney PD, Fetz EE (1980) Functional classes of primate corticomotoneuronal cells and their relation to active force. J Neurophysiol 44:773-791.

Cheney PD, Fetz EE (1985) Comparable patterns of muscle facilitation evoked by individual corticomotoneuronal (CM) cells and by single intracortical microstimuli in primates: evidence for functional groups of CM cells. J Neurophysiol 53:786-804.

Dancause N, Barbay S, Frost SB, Zoubina EV, Plautz EJ, Mahnken JD, Nudo RJ (2006) Effects of small ischemic lesions in the primary motor cortex on neurophysiological organization in ventral premotor cortex. J Neurophysiol 96:3506-3511.

Donoghue JP, Leibovic S, Sanes JN (1992) Organization of the forelimb area in squirrel monkey motor cortex: representation of digit, wrist, and elbow muscles. Exp Brain Res 89:1-19.

Ethier C, Brizzi L, Darling WG, Capaday C (2006) Linear summation of cat motor cortex outputs. J Neurosci 26:5574-5581.

Garcia L, Audin J, D’Alessandro G, Bioulac B, Hammond C (2003) Dual effect of high-frequency stimulation on subthalamic neuron activity. J Neurosci 23:8743-8751.

Garcia L, D’Alessandro G, Fernagut PO, Bioulac B, Hammond C (2005) Impact of high-frequency stimulation parameters on the pattern of discharge of subthalamic neurons. J Neurophysiol 94:3662-3669.

Graziano MS, Taylor CS, Moore T (2002) Complex movements evoked by microstimulation of precentral cortex. Neuron 34:841-851.

Graziano MS, Patel KT, Taylor CS (2004) Mapping from motor cortex to biceps and triceps altered by elbow angle. J Neurophysiol 92:395-407.

Graziano MS, Aflalo TN, Cooke DF (2005) Arm movements evoked by electrical stimulation in the motor cortex of monkeys. J Neurophysiol 94:4209-4223.

Hashimoto T, Elder CM, Okun MS, Patrick SK, Vitek JL (2003) Stimulation of the subthalamic nucleus changes the firing pattern of pallidal neurons. J Neurosci 23:1916-1923.

Hatsopoulos NG, Ojakangas CL, Paninski L, Donoghue JP (1998) Information about movement direction obtained from synchronous activity of motor cortical neurons. Proc Natl Acad Sci U S A 95:15706-15711.

Hendry SH, Jones EG (1981) Sizes and distributions of intrinsic neurons incorporating tritiated GABA in monkey sensory-motor cortex. J Neurosci 1:390-408.

Histed MH, Bonin V, Reid RC (2009) Direct activation of sparse, distributed populations of cortical neurons by electrical microstimulation. Neuron 63:508-522.

Huntley GW, Jones EG (1991) Relationship of intrinsic connections to forelimb movement representations in monkey motor cortex: a correlative anatomic and physiological study. J Neurophysiol 66:390-413.

Jackson A, Gee VJ, Baker SN, Lemon RN (2003) Synchrony between neurons with similar muscle fields in monkey motor cortex. Neuron $38: 115-125$

Keller A (1993) Intrinsic connections between representation zones in the cat motor cortex. Neuroreport 4:515-518.

Keller A, Asanuma H (1993) Synaptic relationships involving local axon collaterals of pyramidal neurons in the cat motor cortex. J Comp Neurol 336:229-242.

Kwan HC, Mackay WA, Murphy JT, Wong YC (1978) An intracortical microstimulation study of output organization in precentral cortex of awake primates. J Physiol (Paris) 74:231-233.

Lemon RN, Muir RB, Mantel GW (1987) The effects upon the activity of hand and forearm muscles of intracortical stimulation in the vicinity of corticomotor neurones in the conscious monkey. Exp Brain Res 66:621-637.

Li S, Arbuthnott GW, Jutras MJ, Goldberg JA, Jaeger D (2007) Resonant 
antidromic cortical circuit activation as a consequence of high-frequency subthalamic deep-brain stimulation. J Neurophysiol 98:3525-3537.

Macpherson JM, Marangoz C, Miles TS, Wiesendanger M (1982) Microstimulation of the supplementary motor area (SMA) in the awake monkey. Exp Brain Res 45:410-416.

Marcus S, Zarzecki P, Asanuma H (1979) An estimate of effective current spread of stimulation current. Exp Brain Res 34:68-71.

Matsumura M, Sawaguchi T, Kubota K (1992) GABAergic inhibition of neuronal activity in the primate motor and premotor cortex during voluntary movement. J Neurophysiol 68:692-702.

McKiernan BJ, Marcario JK, Karrer JH, Cheney PD (1998) Corticomotoneuronal postspike effects in shoulder, elbow, wrist, digit, and intrinsic hand muscles during a reach and prehension task. J Neurophysiol 80:1961-1980.

Naito E, Kinomura S, Geyer S, Kawashima R, Roland PE, Zilles K (2000) Fast reaction to different sensory modalities activates common fields in the motor areas, but the anterior cingulate cortex is involved in the speed of reaction. J Neurophysiol 83:1701-1709.

Nelson RJ, McCandlish CA, Douglas VD (1990) Reaction times for hand movements made in response to visual versus vibratory cues. Somatosens Mot Res 7:337-352.

Park MC (2002) Representation of forelimb muscles in the primary motor cortex of rhesus macaques. Ph.D. thesis, University of Kansas, Molecular and integrative physiology.

Park MC, Belhaj-Saif A, Cheney PD (2000) Chronic recording of EMG activity from large numbers of forelimb muscles in awake macaque monkeys. J Neurosci Methods 96:153-160.

Park MC, Belhaj-Saif A, Gordon M, Cheney PD (2001) Consistent features in the forelimb representation of primary motor cortex in rhesus macaques. J Neurosci 21:2784-2792.

Ranck JB Jr (1975) Which elements are excited in electrical stimulation of mammalian central nervous system: a review. Brain Res 98:417-440.

Sato KC, Tanji J (1989) Digit-muscle responses evoked from multiple intra- cortical foci in monkey precentral motor cortex. J Neurophysiol 62:959-970.

Schieber MH, Deuel RK (1997) Primary motor cortex reorganization in a long-term monkey amputee. Somatosens Mot Res 14:157-167.

Schmidlin E, Wannier T, Bloch J, Rouiller EM (2004) Progressive plastic changes in the hand representation of the primary motor cortex parallel incomplete recovery from a unilateral section of the corticospinal tract at cervical level in monkeys. Brain Res 1017:172-183.

Schmidt EM, McIntosh JS (1990) Microstimulation mapping of precentral cortex during trained movements. J Neurophysiol 64:1668-1682.

Seidemann E, Arieli A, Grinvald A, Slovin H (2002) Dynamics of depolarization and hyperpolarization in the frontal cortex and saccade goal. Science 295:862-865.

Slovin H, Strick P, Hildesheim R, Grinvald A (2003) Voltage sensitive dye imaging in the motor cortex. I. Intra- and intercortical connectivity revealed by microstimulation in the awake monkey. Soc Neurosci Abstr 29:554.8.

Smith WS, Fetz EE (2009a) Synaptic linkages between corticomotoneuronal cells affecting forelimb muscles in behaving primates. J Neurophysiol 102:1040-1048.

Smith WS, Fetz EE (2009b) Synaptic interactions between forelimbrelated motor cortex neurons in behaving primates. J Neurophysiol 102:1026-1039.

Stoney SD Jr, Thompson WD, Asanuma H (1968) Excitation of pyramidal tract cells by intracortical microstimulation: effective extent of stimulating current. J Neurophysiol 31:659-669.

Tolias AS, Sultan F, Augath M, Oeltermann A, Tehovnik EJ, Schiller PH, Logothetis NK (2005) Mapping cortical activity elicited with electrical microstimulation using FMRI in the macaque. Neuron 48:901-911.

Weinrich M, Wise SP (1982) The premotor cortex of the monkey. J Neurosci 2:1329-1345. 